Check for updates

1 The BMJ

2 London

Cite this as: BMJ 2022;376:057 http://dx.doi.org/10.1136/bmj.057 Published: 13 January 2022

\section{Tom Nolan's research reviews-13 January 2022}

\author{
Tom Nolan clinical editorGP', 2
}

\section{Compression chamber}

Training sessions on basic life support always relax a bit once everyone's had a go at chest compressions and debated which song should be sung to get the right tempo. The awkwardness remains but takes on a different quality-like an ambulance siren that sounds different once it's moving away from you. Speaking of blue-light ambulances, should they be going to A\&E or straight to the catheter laboratory after an out-of-hospital cardiac arrest? A multicentre, open-label, randomised trial enrolled 554 patients who had an out-of-hospital cardiac arrest without ST segment elevation to either immediate angiography or initial intensive care assessment (then delayed or selected angiography). ${ }^{1}$ It found no statistically significant difference in death at 30 days, with 54\% dying in the immediate angiography group and $46 \%$ in the delayed angiography group.

\section{I can't get no sleep}

I've managed to repress most memories of hospital night shifts, but the sounds of a ward at night stick in my mind-along with the faces of patients unable to sleep from the sound of clinical waste bins closing, trolley wheels squeaking, and strangers' snores. As well as being another part of the misery of being in hospital, disturbed sleep is a common cause of delirium. Might there be a digital solution to the sleep deprivation of hospital in-patients? Researchers in California devised a decision support tool driven by a "real-time prediction algorithm" to see if identifying inpatients on general wards who could safely forgo four hourly observations overnight would reduce the incidence of delirium. ${ }^{2}$ It didn't, but neither did they see any increase in intensive care admissions or resuscitation calls.

\section{Clotting factors}

When new evidence makes guideline recommendations out of date, it can take years for these to be updated. Living guidelines seek to address this, by updating parts of a guideline as needed. One example is the NICE rapid covid guideline, which has been updated 12 times since it was published in March 2021, but which currently offers no recommendations on thromboprophylaxis in patients discharged from hospital after covid-19. An open-label randomised controlled trial recruited patients being discharged from hospital after covid-19 who were at high risk of venous thromboembolism (VTE). ${ }^{3}$ Three of the 159 people allocated to take rivaroxaban $10 \mathrm{mg}$ for 35 days had a thrombotic event (symptomatic or fatal VTE, asymptomatic VTE, symptomatic arterial thromboembolism, and cardiovascular death), compared with nine of the 159-person control group $(\mathrm{P}=0.0293)$. Although these findings seem promising, I suspect guideline groups will wait for other studies to report before making any recommendations: it's a relatively small study and the rates of VTE were much higher $(5 \%$ of the control group had a symptomatic or fatal VTE) than observed elsewhere (it's typically $0.5-1.6 \%$ in people discharged from hospital after covid-19).

\section{Going with the flow}

The false positive rates of lateral flow tests are back in the spotlight after the change in policy in England so that people who do not have symptoms of covid-19 but do have a positive lateral flow test no longer need to have a confirmatory PCR test. Researchers in Canada reviewed 903408 rapid antigen tests conducted over 537 workplaces between January and October 2021. ${ }^{4}$ A remarkable $278(60 \%)$ of the 462 false positive results occurred in two workplaces and were drawn from a single batch of lateral flow tests. The authors highlight the need to have systems in place that can quickly identify these issues. Fortunately, in the UK we have two systems for logging positive lateral flow tests: the gov.uk website and posting a photo of your positive test on social media along with a sad/shocked/masked face emoji.

\section{Article of no association}

A cohort study in JAMA provides further evidence that use of maternal hormonal contraceptives does not explain a rise in the incidence of CNS tumours in children since the 1970s. This large, prospective, population based study found no association between the two. ${ }^{5}$ It included all live-born children born in Denmark between 1995 and 2014-a total of 1185063 children-thereby winning this week's prize for best population coverage in a research paper.

Competing interests: none declared

Provenance and peer review: commissioned, not peer reviewed

1 Desch S, Freund A, Akin I, etalTOMAHAWK Investigators. Angiography after Out-of-Hospital Cardiac Arrest without ST-Segment Elevation. N Engl J Med 2021;385:2544-53. doi: 10.1056/NEJMoa2101909. pmid: 34459570

2 Najafi N, Robinson A, Pletcher MJ, Patel S. Effectiveness of an Analytics-Based Intervention for Reducing Sleep Interruption in Hospitalized Patients: A Randomized Clinical Trial. JAMA Intern Med 2021. doi: 10.1001/jamainternmed.2021.7387. pmid: 34962506

3 Ramacciotti E, Barile Agati L, Calderaro D, etalMICHELLE investigators. Rivaroxaban versus no anticoagulation for post-discharge thromboprophylaxis after hospitalisation for COVID-19 (MICHELLE): an open-label, multicentre, randomised, controlled trial. Lancet2022;399:50-9. doi: 10.1016/S0140-6736(21)02392-8. pmid: 34921756

4 Gans JS, Goldfarb A, Agrawal AK, Sennik S, Stein J, Rosella L. False-Positive Results in Rapid Antigen Tests for SARS-CoV-2. JAMA 2022. doi: 10.1001/jama.2021.24355. pmid: 34994775

Hargreave M, Mørch LS, Winther JF, Schmiegelow K, Kjaer SK. Association Between Maternal Hormonal Contraception Use and Central Nervous System Tumors in Children. JAMA 2022;327:59-66. doi: 10.1001/jama.2021.22482. pmid: 34982120 